\title{
Occult splenic injury: delayed presentation manifesting as jaundice
}

\author{
R A Simpson, R Ajuwon
}

\begin{abstract}
Delayed splenic rupture, though uncommon, is a recognised clinical condition and is associated with a significant mortality rate. In the vast majority of cases the patient re-presents with abdominal symptoms and/or signs of acute intraperitoneal haemorrhage. A case is presented of delayed splenic rupture presenting with jaundice attributable to intra-abdominal clot lysis.

(Emerg Med f 2001;18:504-505)
\end{abstract}

Keywords: splenic rupture

Case report

A 44 year old man presented to accident and emergency (A\&E) after a riding accident. $\mathrm{He}$ had fallen off the horse over a three feet high jump after which the horse had rolled on to him.

The primary survey revealed a respiratory rate of 20 breaths per minute with normal symmetrical chest expansion, percussion and auscultation. Oxygen saturations were 100\% and his tachypnoea settled with simple analgesia. His pulse rate was 80 beats per minute, blood pressure 175/105 and capillary refill time was two seconds. He scored 15/15 on the Glasgow Coma Scale. Secondary survey revealed slight tenderness over the left posterior chest wall in the infra-scapular region, with no surface bruising. The abdomen was noted to be soft and non-tender. $\mathrm{He}$ denied any abdominal pain. A chest radiograph, taken at the time of the primary survey was thought to be, and subsequently reported as normal. Routine blood tests were normal (haemoglobin, $16.8 \mathrm{~g} / \mathrm{dl}$ ). After a period of stable observation in the department he was discharged home with simple oral analgesia and appropriate advice.

Seven days later the patient re-presented because that day he had become jaundiced and had noticed his urine had become dark brown in colour. Over the preceeding three days he had also noticed a considerable reduction in his exercise tolerance and had been more tired than usual. On close questioning he described a mild left upper quadrant discomfort worsened by deep inspiration. On examination he was comfortable, his abdomen was soft and non-tender, bowel sounds were normal and no masses were palpable. He was haemodynamically stable, and was noted to be clinically jaundiced. Routine blood investigations revealed an haemoglobin of $10.5 \mathrm{~g} / \mathrm{dl}$, and an isolated rise in bilirubin of $35 \mu \mathrm{mol} / 1$.

Computed tomography of the abdomen and pelvis revealed a splenic laceration and haematoma within the spleen. There was a large extra-splenic, sub-diaphragmatic haematoma and free fluid within the abdomen and pelvis. The liver was undamaged. A diagnosis of splenic rupture was made and he was admitted under the duty surgical team. He was managed non-operatively. During the course of his stay he developed a persistant fever. Repeated blood cultures were normal and his fever eventually settled with symptomatic management only. Day 6 after admission he developed a sudden onset of left upper quadrant pain and left shoulder tip pain. Clinical examination revealed tenderness and guarding in the left hypochondrium but the patient remained haemodynamically stable. Full blood count revealed an $\mathrm{Hb}$ of $(9.9 \mathrm{~g} / \mathrm{dl})$. Again he was managed expectantly. The pain had settled the following day and he was eventually discharged home on day 12 after admission on a course of ferrous sulphate.

\section{Discussion}

The spleen is the most commonly injured organ after blunt abdominal trauma. ${ }^{12}$ The vast majority of patients present at the time of injury with symptoms or signs attributable to acute intraperitoneal haemorrhage such as abdominal pain and/or tenderness or hypotension. $^{3}$ Delayed splenic rupture, though relatively uncommon is a recognised clinical entity and is characterised by an asymptomatic period of at least 48 hours between the time of injury and the appearance of signs of internal haemorrhage..$^{4-6}$ The significance of delayed rupture lies in the relatively high mortality rate compared with that associated with acute splenic rupture. ${ }^{7}$ The pathophysiology behind delayed splenic rupture is still not fully understood, but two principal theories predominate. One is that a capsular tear and perisplenic haematoma is formed at the time of injury and is tamponaded by surrounding organs, free rupture into the peritoneal cavity occuring at a later date. The second, more widely accepted theory suggests formation of a subcapsular haematoma at the time of injury; increasing tension develops within it, resulting in subsequent rupture through the capsule and intraperitoneal bleeding and pain. ${ }^{38}$

In conclusion, vigilance and a relatively low threshold for imaging is required in cases of significant abdominal trauma. However, a normal CT scan does not preclude a later splenic rupture and not all cases who present with delayed splenic rupture will have had imaging performed at the time of initial injury. ${ }^{9}$ When delayed splenic rupture does occur the mortality rate is high. ${ }^{10}$ Almost invariably the patient will present with symptoms and/or signs of acute intraperitoneal haemorrhage. ${ }^{3}$ Thankfully, in this case, at the time of 
representation to $\mathrm{A} \& \mathrm{E}$ the patient remained pain free and without clinical signs of abdominal haemorrhage. It was jaundice resulting from clot lysis that brought the patient back to the medical services. To date, we are unaware of any similar cases reported in the literature.

1 Kluger Y, Paul DB, Raves JJ, et al. Delayed rupture of the spleen-myths, facts, and their importance: case reports and literature review. F Trauma 1994;36:568-71.

2 Cox EF. Blunt abdominal trauma. A five year analysis of patients requiring celiotomy. Ann Surg 1984;199:467-4.

3 Davis JJ, Cohn I Jr, Navice FC. Diagnosis and management of blunt abdominal trauma. Ann Surg 1976;183:672-8.
4 Farhat GA, Abdu RA, Vanek VW. Delayed splenic rupture: Real or imaginary? Am Surg 1992;58:340-5.

5 Zabinski EJ, Harkins HN. Delayed splenic rupture: A clinical syndrome following trauma. Arch Surg 1943;46:186213.

6 McIndoe AH. Delayed haemorrhage following traumatic rupture of the spleen. Br f Surg 1931;20:249-68.

7 Webb RC. Traumatic rupture of the normal spleen with delayed haemorrhage. Lancet 1939;59:545-7.

8 Foster RP. Delayed haemorrhage from the ruptured spleen. Br F Surg 1970;57:189.

9 Hertzanu Y, Mendelsohn DB. Delayed splenic rupture: A suggestion for "predictive monitoring". Clin Radiol 1984; 35:393-6.

10 Pappas D, Mirvis SE, Crepps JT. Splenic trauma: false negative $\mathrm{CT}$ diagnosis in cases of delayed rupture. $A \mathcal{F R}$ 1987;149:727

\title{
7th European Forum on Quality Improvement in Health Care
}

\author{
21-23 March 2002 \\ Edinburgh, Scotland
}

We are delighted to announce this forthcoming conference in Edinburgh. Authors are invited to submit papers (call for papers closes on Friday 5 October 2001) and delegate enquiries are welcome.

The themes of the Forum are:

- Leadership, culture change, and change management

- Achieving radical improvement by redesigning care

- Health policy for lasting improvement in health care systems

- Patient safety

- Measurement for improvement, learning, and accountability

- Partnership with patients

- Professional quality: the foundation for improvement

- Continuous improvement in education and training

- People and improvement.

Presented to you by the BMJ Publishing Group (London, UK) and Institute for Healthcare Improvement (Boston, USA). For more information contact: quality@bma.org.uk or look at the website www.quality.bmjpg.com. Tel: +44 (0)20 7383 6409; fax: +44 (0)20 73736869 . 